\title{
Synthesis, Characterization and Antimicrobial Activity of Substituted Semicarbazones
}

\author{
N. SANGEETHA, M. NAGOOR MEERAN* and R. P. SURESHJEYAKUMAR
}

PG \& Research Department of Chemistry, Vivekanandha College of Arts and Sciences for Women (Autonomous), Tiruchengode, Tamilnadu, India

nagoorjmc@rediffmail.com

Received 7 August 2015 / Accpted 2 September 2015

\begin{abstract}
In the present study, a series of substituted semicarbazones were synthesized, characterized and evaluated for their antimicrobial activity. A series of substituted semicarbazones were synthesized from phenyl urea followed by hydrazine hydrate gave good yield of semicarbazide, which were further on treatment with appropriate substituted acetophenones yielded the semicarbazones (B01-B05). All the synthesized compounds were characterized on the basis of their IR, ${ }^{1} \mathrm{H} \&{ }^{13} \mathrm{C}$ NMR and elemental analysis. The antimicrobial activity of all the compounds (B01-B05) showed significant activity against all the bacteria and fungus.
\end{abstract}

Keywords: Semicarbazones, Semicarbazide, Substituted acetophenones, Antimicrobial

\section{Introduction}

Semicarbazones are a class of compounds having the structure $\left[\mathrm{R}_{2} \mathrm{C}=\mathrm{NNHC}(=\mathrm{O}) \mathrm{NH}_{2}\right]^{1}$ formally derived by condensation of aldehydes or ketones with semicarbazide $\left[\mathrm{NH}_{2} \mathrm{NHC}(=\mathrm{O}) \mathrm{NH}_{2}\right]$. They are classified as imine derivatives because they are formed from the reaction of an aldehydes or ketones with the terminal $-\mathrm{NH}_{2}$ group of semicarbazide, which behaves very similarly to primary amines. The substituted semicarbazones possess various biological and pharmacological activities ${ }^{2-11}$. The semicarbazide which are the raw material of semicarbazones have been known to have biological activity against many of the most common species of bacteria ${ }^{12}$. Semicarbazone, themselves are of much interest due to a wide spectrum of anti-fungal and anti-bacterial activities ${ }^{13}$. Recently some researcher had reviewed the bioactivity of semicarbazones and they have exhibited anti-convulsant ${ }^{14}$, antitubercular $^{15}$, anti-oxidant ${ }^{16}$, anti-microbial, analgesic, anti-pyretic ${ }^{17}$ and anti-inflammatory activity $^{18}$. Semicarbazones used as spectrophotometric agents as well for the analysis of metal ions ${ }^{19}$ and are frequently used in the qualitative organic analysis of carbonyl compounds $^{20}$. In view of these data we have undertaken the synthesis, characterization and antimicrobial evaluation of substituted semicarbazones. All the synthesized compounds were characterized on the basis of their physical properties IR, ${ }^{1} \mathrm{H} \&{ }^{13} \mathrm{C}$ NMR spectral data and elemental analysis. The physical data of titled compounds are summarized in Table 1. 
Table 1. Physical and analytical data of the newly synthesized compounds

\begin{tabular}{|c|c|c|c|c|c|c|c|}
\hline \multirow[t]{2}{*}{ Compd. } & \multirow[t]{2}{*}{$\mathrm{R}$} & \multirow{2}{*}{$\begin{array}{l}\text { Yield } \\
\%\end{array}$} & \multirow{2}{*}{$\stackrel{\text { M.P }}{{ }^{0} \mathrm{C}}$} & \multirow{2}{*}{ Mol. Formula } & \multicolumn{3}{|c|}{$\begin{array}{l}\text { Analysis \% found } \\
\text { (Calculated) }\end{array}$} \\
\hline & & & & & $\mathrm{C}$ & $\mathrm{H}$ & $\mathrm{N}$ \\
\hline A03 & - & 60 & 196 & $\mathrm{C}_{7} \mathrm{H}_{6} \mathrm{ClN}_{3} \mathrm{O}_{3}$ & $\begin{array}{l}38.96 \\
(39.02)\end{array}$ & $\begin{array}{c}2.78 \\
(2.69)\end{array}$ & $\begin{array}{l}19.48 \\
(19.35)\end{array}$ \\
\hline H03 & - & 58 & 201 & $\mathrm{C}_{7} \mathrm{H}_{7} \mathrm{ClN}_{4} \mathrm{O}_{3}$ & $\begin{array}{c}36.42 \\
(36.40)\end{array}$ & $\begin{array}{c}3.03 \\
(3.04)\end{array}$ & $\begin{array}{l}24.28 \\
(24.30)\end{array}$ \\
\hline B01 & $\mathrm{H}$ & 61 & 223 & $\mathrm{C}_{15} \mathrm{H}_{13} \mathrm{ClN}_{4} \mathrm{O}_{3}$ & $\begin{array}{c}54.09 \\
(54.10)\end{array}$ & $\begin{array}{c}3.91 \\
(3.95)\end{array}$ & $\begin{array}{l}16.82 \\
(16.84)\end{array}$ \\
\hline B02 & $3-\mathrm{NO}_{2}$ & 63 & 223 & $\mathrm{C}_{15} \mathrm{H}_{12} \mathrm{ClN}_{5} \mathrm{O}_{5}$ & $\begin{array}{c}47.65 \\
(47.66)\end{array}$ & $\begin{array}{c}3.17 \\
(3.19)\end{array}$ & $\begin{array}{l}18.53 \\
(18.55)\end{array}$ \\
\hline B03 & 4- $\mathrm{NO}_{2}$ & 64 & 229 & $\mathrm{C}_{15} \mathrm{H}_{12} \mathrm{ClN}_{5} \mathrm{O}_{5}$ & $\begin{array}{c}47.65 \\
(47.66)\end{array}$ & $\begin{array}{c}3.17 \\
(3.15)\end{array}$ & $\begin{array}{l}18.53 \\
(18.50)\end{array}$ \\
\hline B04 & $3-\mathrm{NH}_{2}$ & 58 & 240 & $\mathrm{C}_{15} \mathrm{H}_{14} \mathrm{ClN}_{5} \mathrm{O}_{3}$ & $\begin{array}{c}51.76 \\
(51.70)\end{array}$ & $\begin{array}{c}4.03 \\
(4.00)\end{array}$ & $\begin{array}{l}20.12 \\
(20.10)\end{array}$ \\
\hline B05 & $4-\mathrm{NH}_{2}$ & 56 & 256 & $\mathrm{C}_{15} \mathrm{H}_{14} \mathrm{ClN}_{5} \mathrm{O}_{3}$ & $\begin{array}{l}51.76 \\
(51.75)\end{array}$ & $\begin{array}{c}4.03 \\
(4.02)\end{array}$ & $\begin{array}{l}20.12 \\
(20.11)\end{array}$ \\
\hline
\end{tabular}

\section{Experimental}

The melting points were carried out in open capillary tube and were uncorrected. Thin layer chromatography was performed using silica gel coated on a glass plate and spots were visualized by exposure to iodine vapor. IR spectra of compounds were scanned on Shimadzu IR spectrophotometer using $\mathrm{KBr}$ disc and expressed in $\mathrm{cm}^{-1} .{ }^{1} \mathrm{H} \&{ }^{13} \mathrm{C}$ NMR spectra were recorded in DMSO-D ${ }_{6}$ on BRUKER $(400 \mathrm{MHz})$ spectrometer using TMS as an internal standard (chemical shifts in $\delta, \mathrm{ppm}$ ) (Table 2). The elemental analysis for $\mathrm{C}, \mathrm{H} \& \mathrm{~N}$ were in an agreement with the calculated values. The synthesis of the target compounds was accomplished according to the reaction sequence illustrated in Scheme 1.
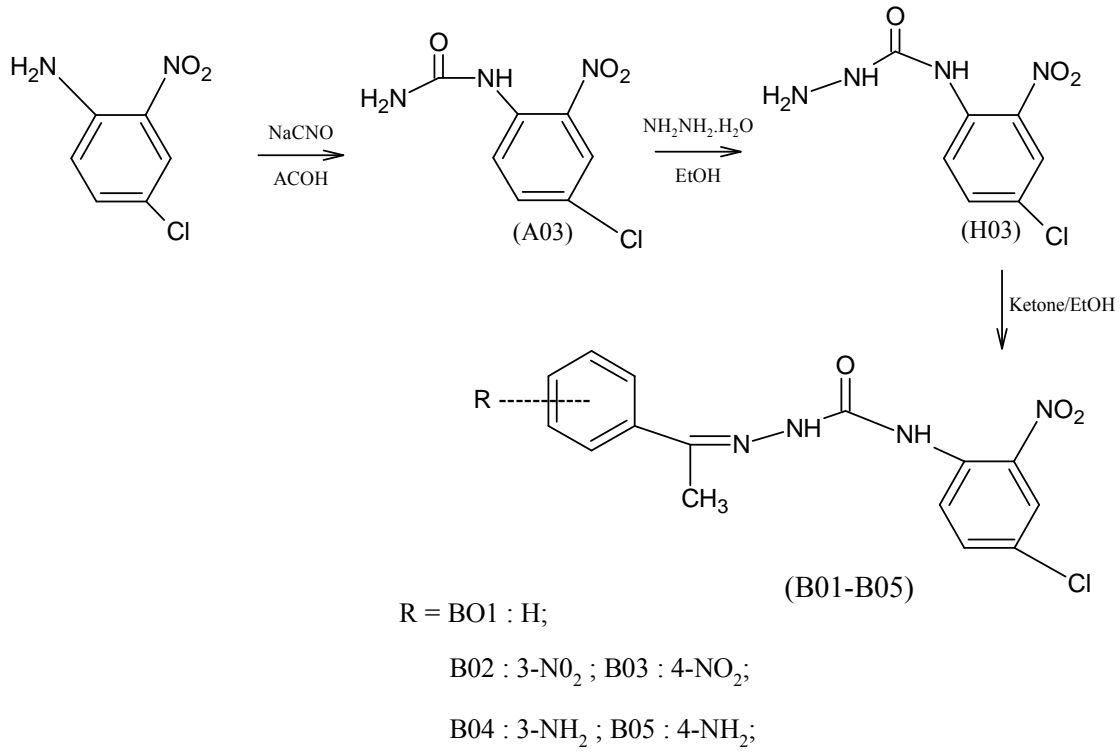

Scheme 1 
Table 2. Spectral data of synthesized compounds

\begin{tabular}{|c|c|c|c|}
\hline Comp. & $\operatorname{IR}(\mathrm{KBr}) v_{\max }$ in $\mathrm{cm}^{-1}$ & $\begin{array}{c}{ }^{1} \mathrm{H} \text { NMR }\left(\text { DMSO }^{\text {ppm }}\right), \delta \\
\end{array}$ & $\begin{array}{c}{ }^{13} \mathrm{C} \text { NMR }\left(\mathrm{DMSO}^{1} \mathrm{~d}_{6}\right), \\
\delta, \mathrm{ppm}\end{array}$ \\
\hline A03 & $\begin{array}{l}3471 \text { (NH str), } 3093 \\
\text { (aromatic C-H str ), } 1635 \\
(\mathrm{C}=\mathrm{O} \text { str), } 1566 \text { (aromatic } \\
\mathrm{C}=\mathrm{C}), 1249\left(\mathrm{NO}_{2} \text { str) }\right.\end{array}$ & $\begin{array}{l}6.53\left(\mathrm{~s}, 2 \mathrm{H}, \mathrm{CONH}_{2}\right), \\
8.98(\mathrm{~s}, 1 \mathrm{H}, \text { Ar-NH }), \\
6.5-8.6(\mathrm{~m}, 3 \mathrm{H}, \text { Ar-H })\end{array}$ & $\begin{array}{l}168(\mathrm{CONH}), 117-145 \\
(\mathrm{Ar}-\mathrm{C})\end{array}$ \\
\hline H03 & $\begin{array}{l}3471 \text { (NH str), } 3093 \\
\text { (aromatic C-H str), } 1635 \\
(\mathrm{C}=\mathrm{O} \text { str), } 1566 \text { (aromatic } \\
\mathrm{C}=\mathrm{C}), 1249\left(\mathrm{NO}_{2} \text { str) }\right.\end{array}$ & $\begin{array}{l}4.53\left(\mathrm{~s}, 2 \mathrm{H}, \mathrm{NH}_{2}\right), 8.17 \\
(\mathrm{~s}, 1 \mathrm{H}, \mathrm{CONH}), 8.19(\mathrm{~s}, \\
1 \mathrm{H}, \mathrm{Ar}-\mathrm{NH}), 6.92-8.15 \\
(\mathrm{~m}, 3 \mathrm{H}, \mathrm{Ar}-\mathrm{H})\end{array}$ & $\begin{array}{l}168(\mathrm{CONH}), 118- \\
145(\mathrm{Ar}-\mathrm{C})\end{array}$ \\
\hline B01 & $\begin{array}{l}3471 \text { (NH str), } 3093 \text { (C-H } \\
\text { str), } 2854 \text { (aliphatic C-H } \\
\text { str), } 1635 \text { (C=O str), } 1597 \\
(\mathrm{C}=\mathrm{N} \text { str), } 1566 \text { (aromatic } \\
\mathrm{C}=\mathrm{C}) \text { and } 1249\left(\mathrm{NO}_{2} \text { str) }\right.\end{array}$ & $\begin{array}{lll}2.269 & (\mathrm{~s}, \quad 3 \mathrm{H}, & \left.\mathrm{CH}_{3}\right), \\
7.039 & (\mathrm{~s}, 1 \mathrm{H}, & \mathrm{Ar}-\mathrm{NH}), \\
7.061 & -7.937 \quad(\mathrm{~m}, & 8 \mathrm{H}, \\
\text { Ar-H), } 11.26 \quad(\mathrm{~s}, & 1 \mathrm{H}, \\
\text { CONH) } & \end{array}$ & $\begin{array}{l}184 \quad(\mathrm{CONH}), \quad 166 \\
(\mathrm{Ph}-\mathrm{C}=\mathrm{N}), \quad 113-150 \\
(\mathrm{Ar}-\mathrm{C}), 26\left(\mathrm{CH}_{3}\right)\end{array}$ \\
\hline B02 & $\begin{array}{l}3471 \text { (NH str), } 3093 \text { (C-H } \\
\text { str }), 2854 \text { (aliphatic C-H } \\
\text { str), } 1635 \text { (C=O str), } 1597 \\
(\mathrm{C}=\mathrm{N} \text { str), } 1566 \text { (aromatic } \\
\mathrm{C}=\mathrm{C}) \text { and } 1249\left(\mathrm{NO}_{2} \text { str) }\right.\end{array}$ & $\begin{array}{l}2.19\left(\mathrm{~s}, 3 \mathrm{H}, \mathrm{CH}_{3}\right), 8.404 \\
(\mathrm{~s}, 1 \mathrm{H}, \mathrm{Ar}-\mathrm{NH}), 7.055- \\
8.388(\mathrm{~m}, 7 \mathrm{H}, \quad \mathrm{Ar}-\mathrm{H}), \\
11.13(\mathrm{~s}, 1 \mathrm{H}, \mathrm{CONH})\end{array}$ & $\begin{array}{l}183 \quad(\mathrm{CONH}), \quad 169 \\
(\mathrm{Ph}-\mathrm{C}=\mathrm{N}), \quad 118-148 \\
(\mathrm{Ar}-\mathrm{C}), 26.9\left(\mathrm{CH}_{3}\right)\end{array}$ \\
\hline B03 & $\begin{array}{l}3471 \quad(\mathrm{NH} \text { str), } \\
\text { (aromatic } \mathrm{C}-\mathrm{H} \text { str), } 2854 \\
\text { (aliphatic } \mathrm{C}-\mathrm{H} \text { str), } 1635 \\
(\mathrm{C}=\mathrm{O} \text { str), } 1597 \quad(\mathrm{C}=\mathrm{N} \\
\text { str), } 1566 \text { (aromatic } \mathrm{C}=\mathrm{C}) \\
\text { and } 1249\left(\mathrm{NO}_{2} \text { str) }\right.\end{array}$ & $\begin{array}{l}2.282\left(\mathrm{~s}, 3 \mathrm{H}, \mathrm{CH}_{3}\right), \\
8.361(\mathrm{~s}, 1 \mathrm{H}, \quad \mathrm{Ar}-\mathrm{NH}), \\
7.05-8.34(\mathrm{~m}, 7 \mathrm{H}, \mathrm{Ar}- \\
\mathrm{H}), 11.287 \quad(\mathrm{~s}, \quad 1 \mathrm{H}, \\
\text { CONH })\end{array}$ & $\begin{array}{l}183(\mathrm{CONH}), 167(\mathrm{Ph}- \\
\mathrm{C}=\mathrm{N}), 118-149 \quad(\mathrm{Ar}- \\
\mathrm{C}), 27\left(\mathrm{CH}_{3}\right)\end{array}$ \\
\hline B04 & $\begin{array}{l}3471 \quad(\mathrm{NH} \text { str), } \\
\text { (aromatic C-H str), } 2854 \\
\text { (aliphatic C-H str), } 1635 \\
(\mathrm{C}=\mathrm{O} \text { str), } 1597 \quad(\mathrm{C}=\mathrm{N} \\
\text { str), } 1566 \text { (aromatic } \mathrm{C}=\mathrm{C}) \\
\text { and } 1249\left(\mathrm{NO}_{2} \text { str) }\right.\end{array}$ & $\begin{array}{l}2.197\left(\mathrm{~s}, \quad 3 \mathrm{H}, \mathrm{CH}_{3}\right), \\
4.911\left(\mathrm{~s}, 2 \mathrm{H}, \mathrm{Ar}-\mathrm{NH}_{2}\right), \\
7.63(\mathrm{~s}, 1 \mathrm{H}, \mathrm{Ar}-\mathrm{NH}), \\
6.91-7.62(\mathrm{~m}, 7 \mathrm{H}, \mathrm{Ar}- \\
\mathrm{H}), 11.13 \quad(\mathrm{~s}, \quad 1 \mathrm{H}, \\
\text { CONH) }\end{array}$ & $\begin{array}{l}184(\mathrm{CONH}), 164(\mathrm{Ph}- \\
\mathrm{C}=\mathrm{N}), 111-159 \quad(\mathrm{Ar}- \\
\mathrm{C}), 22\left(\mathrm{CH}_{3}\right)\end{array}$ \\
\hline B05 & 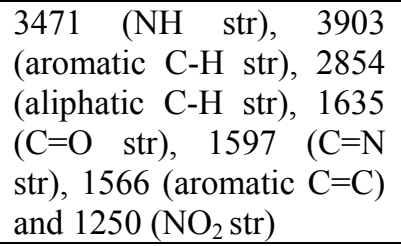 & $\begin{array}{l}2.258\left(\mathrm{~s}, 3 \mathrm{H}, \mathrm{CH}_{3}\right), \\
4.52\left(\mathrm{~s}, 2 \mathrm{H}, \mathrm{Ar}-\mathrm{NH}_{2}\right), \\
7.93(\mathrm{~s}, 1 \mathrm{H}, \mathrm{Ar}-\mathrm{NH}), \\
6.77-7.87(\mathrm{~m}, 7 \mathrm{H}, \mathrm{Ar}- \\
\mathrm{H}), 11.01 \quad(\mathrm{~s}, \quad 1 \mathrm{H}, \\
\text { CONH) }\end{array}$ & $\begin{array}{l}186 \quad(\mathrm{CONH}), \quad 163 \\
(\mathrm{Ph}-\mathrm{C}=\mathrm{N}), \quad 108-145 \\
(\mathrm{Ar}-\mathrm{C}), 23\left(\mathrm{CH}_{3}\right)\end{array}$ \\
\hline
\end{tabular}

\section{General procedure ${ }^{21}$}

Synthesis of 1-(4-chloro-2-nitrophenyl) urea (A03)

The substituted aniline $(8.625 \mathrm{~g}, 0.05 \mathrm{~mole})$ was dissolved in glacial acetic acid $(20 \mathrm{~mL})$ and diluted with water $(100 \mathrm{~mL})$. To this, an equimolar quantity $(3.25 \mathrm{~g}, 0.05$ mole $)$ of sodium cyanate in warm water $(50 \mathrm{~mL})$ was added with constant (45 minuts) stirring. The reactionmixture was allowed to stand for $1 \mathrm{~h}$ and the solid precipitate formed was filtered off and dried after recrystalization from boiling water. 


\section{Synthesis of N-(4-chloro-2-nitrophenyl) hydrazinecarboxamide (H03)}

To a solution of 1-(4-chloro-2-nitrophenyl)urea(A03) (2.155 g, $0.01 \mathrm{~mole})$ in ethanol $(20 \mathrm{~mL})$, an equimolar quantity of hydrazine hydrate $(0.6 \mathrm{~g})$ was added. The reaction mixture was made alkaline by adding sodium hydroxide $(1 \mathrm{~g})$. The reaction mixture was refluxed for $6 \mathrm{~h}$ and the precipitate obtained after cooling and filtered then washed and dried. Recrystallized from ethanol.

\section{Synthesis of substituted Semicarbazones (B01-B05)}

A mixture of substituted phenyl semicarbazide(H03) (0.01 mole) and appropriate substituted acetophenones $(0.01 \mathrm{~mole})$ in ethanol $(25 \mathrm{~mL})$ was refluxed, in the presence of few drops of glacial acetic acid. After $1 \mathrm{~h}$, the precipitate obtained was filtered and recrystallized from ethanol.

\section{Antimicrobial activity}

In vitro antibacterial activity was determined by Kirby-Bauer disc diffusion method against bacteria such as Staphylococcus aureus and Bacillus (Gram +ve), Salmonella typhi and Pseudomonas aeruginosa (Gram -ve) using Ampicilin as standard a standard drug. The standard and test compounds were prepared in DMSO at different concentrations. The zone of inhibition was compared with standard drug after $24 \mathrm{~h}$ incubation at $35-37{ }^{\circ} \mathrm{C}$.

Similarly antifungal activity was performed against Candida. The standard and test compounds were prepared in DMSO at different concentrations. The zone of inhibition was compared with standard drug after $48 \mathrm{~h}$ at $25{ }^{\circ} \mathrm{C}$. The results of antibacterial and antifungal activity are presented in Table 3.

Table 3. Antimicrobial activity of the synthesized compounds zone of inhibition (mm) of synthesized compounds

\begin{tabular}{|c|c|c|c|c|c|c|c|c|c|c|c|c|c|c|c|c|c|c|c|}
\hline \multirow{4}{*}{$\begin{array}{l}\frac{0}{0} \\
0 \\
0 \\
\tilde{0} \\
\tilde{\Xi} \\
\tilde{D}\end{array}$} & \multicolumn{16}{|c|}{ Anti-bacterial activity } & \multirow{2}{*}{\multicolumn{3}{|c|}{$\begin{array}{c}\text { Anti-fungal } \\
\text { activity }\end{array}$}} \\
\hline & \multicolumn{8}{|c|}{ Gram positive } & \multicolumn{8}{|c|}{ Gram negative } & & & \\
\hline & \multicolumn{4}{|c|}{$\begin{array}{c}\text { Staphylococcus } \\
\text { Spp. } \\
\end{array}$} & \multicolumn{4}{|c|}{ Bacillus spp. } & \multicolumn{4}{|c|}{ Salmonella spp. } & \multicolumn{4}{|c|}{$\begin{array}{c}\text { Pseudomonas } \\
\text { Spp. }\end{array}$} & \multicolumn{3}{|c|}{ Candida } \\
\hline & $\begin{array}{l}\text { b } \\
\circlearrowright \\
\Xi \\
8 \\
\varnothing\end{array}$ & $\begin{array}{l}\stackrel{0}{0} \\
\stackrel{\Xi}{\Xi} \\
0 \\
0\end{array}$ & 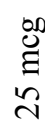 & in & $\begin{array}{l}8 \\
0 \\
\Xi \\
8 \\
8\end{array}$ & 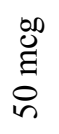 & 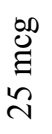 & $\vec{D}$ & $\begin{array}{l}00 \\
0 \\
\Xi \\
8 \\
8\end{array}$ & 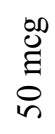 & $\begin{array}{l}e_{0}^{\infty} \\
\Xi \\
\end{array}$ & 离 & $\begin{array}{l}0 \\
0 \\
\Xi \\
8 \\
8\end{array}$ & 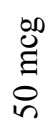 & $\begin{array}{l}\stackrel{00}{0} \\
\Xi \\
\end{array}$ & $\stackrel{\vec{D}}{\mathscr{D}}$ & $\begin{array}{l}0 \\
0 \\
\Xi \\
8 \\
8\end{array}$ & 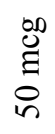 & 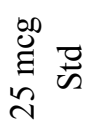 \\
\hline B01 & 8 & 4 & - & 10 & 5 & 3 & - & 10 & 5 & 3 & - & 9 & 6 & 4 & 3 & 9 & 5 & 4 & -14 \\
\hline B02 & 9 & 4 & 3 & 11 & 8 & 6 & 3 & 12 & 6 & 4 & 3 & 10 & 9 & 8 & 5 & 5 & 5 & 3 & -15 \\
\hline B03 & 6 & 3 & - & 5 & 7 & 4 & 3 & 7 & 8 & 5 & 3 & 5 & 8 & 7 & 5 & 10 & 6 & 5 & -12 \\
\hline B04 & 6 & 5 & 3 & 7 & 9 & 7 & 5 & 9 & 9 & 8 & 7 & 10 & 7 & 5 & 4 & 8 & 5 & 4 & -21 \\
\hline B05 & 8 & 6 & 4 & 9 & 9 & 7 & 5 & 9 & 9 & 8 & 7 & 9 & 9 & 8 & 6 & 12 & 5 & 3 & -20 \\
\hline
\end{tabular}

\section{Results and Discussion}

Synthesis of substituted semicarbazones was obtained as mentioned in the Scheme 1. The required starting material phenyl urea (A03) was synthesized according to the procedure reported in the literature. Hydrazinolysis of the urea with hydrazine hydrate gave good yield of semicarbazides, which were further on treatment with appropriate carbonyl compound yielded the semicarbazones (B01-B05). The spectral analysis of all the compounds was done by IR, ${ }^{1} \mathrm{H}$ and ${ }^{13} \mathrm{C}$ NMR and the spectral data were consistent with the assigned structures. 
In vitro antibacterial activity of selected compounds was carried out by Kirby-Bauer disc diffusion method against bacteria such as Staphylococcus aureus, Bacillus, Salmonella typhi, Pseudomonas aeruginosa. The compounds B01-B05 showed significant activity against all the bacteria. Antifungal activity was performed on candida. The compounds B01, B02, B04 and B05 showed moderate activity against the fungus. The compound B03 was more active among screened compounds.

\section{Conclusion}

The reaction profile explained in the present work is very efficient to synthesized substituted semicarbazones. The prepared compounds showed potent antimicrobial activities and these are promising compounds for further pharmacological studies.

\section{References}

1. Dimmock J R, Puthucode R N, Tuchek J, Baker G B,.Hinko C N, Steinmiller C L and Stables J P, Drug Dev Res., 1999, 46(2), 112-125; DOI:10.1002/(SICI)10982299(199902)46:2<112::AID-DDR4>3.0.CO;2-N

2. AlamV, Mallick P, Verma S P, Gilani S J, Khan S A, Siddiqui N and Ahsan W, Eur J Med Chem., 2010, 45(6), 2467-2472; DOI:10.1016/j.ejmech.2010.02.031

3. Afrasiabi Z, Sinn E K K, Lin W, Ma Y, Campana C and Padhye S, J Inorg Biochem., 2005, 99(7), 1526-1531; DOI:10.1016/j.jinorgbio.2005.04.012

4. Dutta S, Padhye S, Priyadarsini K I and Newton C, Bioorg Med Chem Lett., 2005, 15(11), 2738-2744; DOI:10.1016/j.bmcl.2005.04.001

5. Noblia P, Vieites M, Costa B S P, Baran E J, Cerecetto H, Draper P, Gonzalez M, Piro O E, Castellano E E, Azueta A, Cerain A L D, Monge-vega A and Gambino D, J Inorg Bio., 2005, 99(2), 443-451; DOI:10.1016/j.jinorgbio.2004.10.019

6. Mishra V, Pandeya S N, Declercq E, Pannecoque C and Witvrouw M, Pharmaceut Acta Helvet., 1998, 73(4), 215-218; DOI:10.1016/S0031-6865(98)00028-4

7. Cerecetto H, Maio R D, Gonzalez M, Risso M, Sagrera G, Seoane G, Denicola A, Peluffo G, Quijano C, Stoppani A O M, Paulino M, Olea-Azar C and Basombrio M A, Eur J Med Chem., 2000, 35(3), 343-350; DOI:10.1016/S0223-5234(00)00131-8

8. Cerecetto H, Maio R D, Ibarruri G, Seoane G, Denicola A, Peluffo A, Quijano C and Paulino M, IL Farmaco, 1998, 53(2), 89-94; DOI:10.1016/S0014-827X(97)00011-6

9. Chikar N, Kasuga A, Sekino K, Koumo C, Shimada N, Ishikawa M and Nomoiya K, J Inorg Biochem., 2001, 84(1-2), 55-65; DOI:10.1016/S0162-0134(00)00221-X

10. Bernard S, Pillat C, Oddos T, Seman M and Milcent R, Eur J Med Chem., 1995, 30(6), 471-482; DOI:10.1016/0223-5234(96)88258-4

11. Martinez- Merino V and Cerecetto H, Bioorg Med Chem., 2001, 9(4), 1025-1030; DOI:10.1016/S0968-0896(00)00320-5

12. Dorgan H N, Duran A andYemini E, Drug Metabol Drug Interact., 1999, 15, 187-95.

13. Singhal M and Paul A, Int J Pharm Sci Res., 2011a, 2, 2602-04.

14. Pandeya S N, Yogeeswari P and Stable J P, Euro J Med Chem., 2000, 35(10), 879886; DOI:10.1016/S0223-5234(00)01169-7

15. Sriram D, Yogeeswari P and Thirumurugan R, Bioorg Med Chem Lett., 2000, 14(15), 3923-3924; DOI:10.1016/j.bmcl.2004.05.060

16. Singhal M and Paul A, Global J Pharmacol., 2011b, 5, 60-66.

17. Singhal M and Paul A, Res J Pharmacol., 2011c, 5, 47-52. 
18. Singh H P, Chauhan C S, Pandeya S N, Sharma C S, Srivastava, Der PharmaLett 2010, 2, 460-472.

19. Atalay T and Akgemci E G, Tur J Chem., 1998, 22(2), 123-127.

20. Kolb V M, Stupar J W, Janota T E and Duax W L, J Org Chem., 1989, 54(10), 23412346; DOI:10.1021/jo00271a019

21. Raja A S, Agarwal A K, Mahajan N, Pandeya S N and Ananthan S, Indian J Chem Sec B, 2010, 49B, 1384-1388. 